\title{
Modeling the AgInSbTe Memristor
}

\author{
Juntang $Y U^{1}$, Yi $L^{2}{ }^{2}$, Xiaomu $M U^{1}$, Jinjian $Z H A N G^{2}$, Xiangshui MIAO ${ }^{2}$, Shuning WANG $^{1}$ \\ ${ }^{1}$ Dept. of Automation, Tsinghua National Laboratory for Information Science and Technology (TNList), \\ Tsinghua University, Qinghuayuan 1, 100084 Beijing, People's Republic of China \\ ${ }^{2}$ Wuhan National Laboratory for Optoelectronics, School of Optical and Electronic Information, Huazhong University of \\ Science and Technology, 430074 Wuhan, People's Republic of China
}

yjt11@mails.tsinghua.edu.cn, liyi@hust.edu.cn, mxm09@mails.tsinghua.edu.cn, zhangjinjian1221@gmail.com, miaoxs@hust.edu.cn, swang@mail.tsinghua.edu.cn

\begin{abstract}
The AgInSbTe memristor shows gradual resistance tuning characteristics, which makes it a potential candidate to emulate biological plastic synapses. The working mechanism of the device is complex, and both intrinsic charge-trapping mechanism and extrinsic electrochemical metallization effect are confirmed in the AgInSbTe memristor. Mathematical model of the AgInSbTe memristor has not been given before. We propose the flux-voltage controlled memristor model. With piecewise linear approximation technique, we deliver the flux-voltage controlled memristor model of the AgInSbTe memristor based on the experiment data. Our model fits the data well. The flux-voltage controlled memristor model and the piecewise linear approximation method are also suitable for modeling other kinds of memristor devices based on experiment data.
\end{abstract}

\section{Keywords}

Memristor, mathematical model, piecewise linear

\section{Introduction}

Memristor is characterized by a relation between the flux $\varphi$ and the charge $q$, and is proposed as the fourth basic circuit element, which is as basic as the resistor, the inductor and the capacitor [1]. According to [1], [2], an ideal flux controlled memristor is defined as

$$
\mathrm{d} q=W(\varphi(t)) \mathrm{d} \varphi
$$

where $W(\varphi(t))$ is the conductance of memristor. The current $i(t)$ and voltage $v(t)$ relation is described by (note that $\mathrm{d} q=i \mathrm{~d} t$ and $\mathrm{d} \varphi=v \mathrm{~d} t)$

$$
i(t)=W(\varphi(t)) v(t)
$$

Similarly, an ideal charge controlled memristor is defined as

$$
\mathrm{d} \varphi=M(q(t)) \mathrm{d} q,
$$

where $M(q(t))$ is the resistance of memristor. The current $i(t)$ and voltage $v(t)$ relation is described by

$$
v(t)=M(q(t)) i(t)
$$

Memristive system is a generalization of the memristor [3], [4]. According to [3], a voltage controlled memristive system is described by

$$
\begin{aligned}
\dot{x} & =f(x, v, t), \\
i & =G(x, v, t) v,
\end{aligned}
$$

where $x$ is the state variable of the memristive system, and $f(\cdot)$ and $G(\cdot)$ are functions of $x, v$ and $t$. The ideal memristor is a special case of the memristive system (letting $x=\varphi$, $f(x, v, t)=\dot{\varphi}=v$ and $G(x, v, t)=W(\varphi(t)))$. Similarly, a current controlled memristive system is described by

$$
\begin{aligned}
& \dot{x}=h(x, i, t), \\
& v=R(x, i, t) i,
\end{aligned}
$$

where $h(\cdot)$ and $R(\cdot)$ are functions of $x, i$ and $t$.

The first physical memristor device was found by [5]. The two-terminal electrical device is a thin semiconductor film sandwiched between two metal contacts. The semiconductor film has two regions: one region with a high dopant concentration and low resistance, and the other region with a low concentration of dopant and much higher resistance. The charges that pass through the device will move the boundary of the two regions, and therefore change the total resistance of the device. More detailed description of the device can be found in [5]. Then many other physical memristor devices with different physical mechanisms have been proposed by researchers, such as [6], [7], [8].

To further study and apply a memristor device, a suitable model is necessary. The ideal memristor definition (1) and (3) usually cannot fully describe the complex nature of the physical memristor devices, so the memristive system is a common choice of modeling physical memristor devices [5], [9], [10], [11]. There are models of a particular memristor device [7], [9]. In [7], the following model is given to model the tantalum oxide memristors, 


$$
\begin{gathered}
i=v\left[y G_{m}+(1-y) a \exp (b \sqrt{|v|})\right] \\
\frac{\mathrm{d} y}{\mathrm{~d} t}=\left\{\begin{array}{c}
B \sinh \left[v / \sigma_{O N}\right] \exp \left[-\left(y / y_{O N}\right)^{2}\right] \exp \left[p / \sigma_{p}\right] \\
v>0, \\
A \sinh \left[v / \sigma_{O F F}\right] \exp \left[-\left(y / y_{O F F}\right)^{2}\right] \exp [1 /(1+\beta p)] \\
v<0,
\end{array}\right.
\end{gathered}
$$

where state variable $y$ is the volume fraction (equivalently cross-sectional area) of the channel with metallic transport, $p$ is the power, $G_{m}, a, b, B, A, \sigma_{O N}, \sigma_{O F F}, y_{O N}, y_{O F F}, \sigma_{p}, \beta$ are constants [7]. To describe different types of memristor devices, many flexible general models are proposed [10], [11], [12]. As an example, general model proposed by [10] is given by

$$
\begin{aligned}
& i(t)= \begin{cases}\alpha_{1} x(t) \sinh (b v(t)) & v(t)>0, \\
\alpha_{2} x(t) \sinh (b v(t)) & v(t)<0,\end{cases} \\
& \frac{\mathrm{d} x}{\mathrm{~d} t}=g(v(t)) f(x(t)),
\end{aligned}
$$

where $\alpha_{1}, \alpha_{2}, b$ are parameters, and $g(v(t)), f(x(t))$ are nonlinear functions with parameters. Different types of physical memristor devices have different parameters in (8). This model is suitable for device responses for sinusoidal and linearly increasing inputs [10].

In this paper, we focus on the AgInSbTe memristor proposed in [8]. The AgInSbTe memristor shows gradual resistance tuning characteristics and good potential in emulating biological plastic synapses. To our best knowledge, unlike model (7) for the tantalum oxide memristors, no mathematical model of the AgInSbTe memristor has been given in published papers. We propose a new model of memristors and use it to model the AgInSbTe memristor with the experiment data. The piecewise linear technique is used in the model, which allows the parameter fitting to be done with existing effective identification algorithms. The piecewise linear approximation can achieve a good precision. Simulation results show that our model fits the experiment data well. Gradual resistance tuning and other characteristics of the AgInSbTe memristor can be fully modeled. Our model is also suitable for modeling other different types of physical memristor devices.

\section{Flux-Voltage Controlled Memristor Model}

Voltage dependence is observed in many physical memristor devices [7], [9], [11], including the AgInSbTe memristor we study in this paper. The flux controlled memristor (1),(2) cannot model the voltage dependence of the physical memristor devices, since the conductance $W(\varphi(t))$ is only determined by the history of $v(t)$, i.e. $\varphi(t)=\int v(\tau) \mathrm{d} \tau$, not the present $v(t)$. Here we propose the flux-voltage controlled memristor model:

$$
\begin{aligned}
i(t) & =\frac{1}{R(\varphi(t), v(t))} v(t), \\
\frac{\mathrm{d} \varphi(t)}{\mathrm{d} t} & =v(t) .
\end{aligned}
$$

$R(\varphi(t), v(t))$ is the resistance of the memristor device. The flux-voltage controlled memristor model is a natural extension of the flux controlled memristor (2), and has taken the voltage into consideration. It also fits the memristive system definition (5), where the flux $\varphi(t)$ is the state variable. Thus, the flux-voltage controlled memristor can be seen as a kind of special case of the memristive system.

The key to model the AgInSbTe memristor is getting an analytic expression of $R(\varphi(t), v(t))$ that fits the experiment data well. Here the piecewise linear technique is applied to achieve it. Piecewise and piecewise linear techniques have long been used to model and analyze nonlinear electronic circuit [13], including the memristor [14], [15], [16]. For example, piecewise linear flux-charge relationship was proposed in [14]. In [17], piecewise linear window function was used to model the physical memristor device. An image encryption algorithm based on piecewise linear memristor was given in [18]. Piecewise linear function has strong approximation capability and corresponding effective identification algorithms [13], [19], [20].

Particularly, we use the hinging hyperplane (HH) [19] in this paper. $R(\varphi(t), v(t))$ represented by $\mathrm{HH}$ functions is given by

$$
\begin{aligned}
R(\varphi(t), v(t))= & a_{0} \varphi(t)+b_{0} v(t)+c_{0} \\
& +\sum_{i=1}^{m} \eta_{i} \max \left\{0, a_{i} \varphi(t)+b_{i} v(t)+c_{i}\right\}
\end{aligned}
$$

where $a_{i}, b_{i}, c_{i}, i=0,1, \ldots, m$ and $\eta_{i}, i=1,2, \ldots, m$ are the parameters. In (10), $\max \left\{0, a_{i} \varphi(t)+b_{i} v(t)+c_{i}\right\}$ is the hinge function. $\max \left\{0, a_{i} \varphi(t)+b_{i} v(t)+c_{i}\right\}$ is equal to different linear functions 0 or $a_{i} \varphi(t)+b_{i} v(t)+c_{i}$ at different subregions of the domain $\left(a_{i} \varphi(t)+b_{i} v(t)+c_{i}<0\right.$ and $\left.a_{i} \varphi(t)+b_{i} v(t)+c_{i}>0\right)$, separated by the hinge $a_{i} \varphi(t)+$ $b_{i} v(t)+c_{i}=0$. The HH functions have strong capability in approximating nonlinear functions [19]. For simple examples with only 2 hinge functions, assume $\alpha<\beta$, $-\max \{0,-x+\alpha\}+\max \{0, x-\beta\}$ has threshold characteristic on $[\alpha, \beta], \max \{0, x-\alpha\}-\max \{0, x-\beta\}$ has saturation characteristic on $(-\infty, \alpha]$ and $[\beta,+\infty)$. Nonlinear characteristics, including threshold and saturation are often observed in physical memristor devices [7], [11].

For the ideal charge controlled memristor and the current controlled memristive system, we similarly propose the charge-current controlled memristor model:

$$
\begin{aligned}
v(t) & =R(q(t), i(t)) i(t), \\
\frac{\mathrm{d} q(t)}{\mathrm{d} t} & =i(t) .
\end{aligned}
$$


When $R(q(t), i(t))$ is represented by $\mathrm{HH}$ functions, a hinge function takes the form of $\max \left\{0, a_{i} q(t)+b_{i} i(t)+c_{i}\right\}$.

It should be pointed out that the flux-voltage controlled model is different from the memristor model with neural network of smooth hinge functions proposed in [21]. The resistance function $R(\varphi(t), v(t))$ in our model is piecewise linear, while that in the other model is not. The analysis of memristor circuits can be transformed into piecewise linear optimization problems when our model is applied. There are many effective algorithms to deal with them [22], [23], [24].

\section{Modeling the AgInSbTe Memristor}

As mentioned in Sec. 2, the ideal flux controlled memristor (1), (2) cannot model the voltage dependence of the AgInSbTe memristor well. The co-existence of intrinsic charge-trapping mechanism and extrinsic electrochemical metallization effect is confirmed in the AgInSbTe memristor [8] and oxide memristors [25]. The mechanism of the device is complex. It is not easy to choose a particular state variable of the device that has physical meaning and is measurable, such as volume fraction $y$ in (7), when using the general voltage controlled memristive system (5) to model the AgInSbTe memristor.

With the experiment data given in [8], the flux-voltage controlled memristor model (9) of AgInSbTe memristor can be obtained by finding a suitable $R(\varphi(t), v(t))$ for different kinds of input signals. Considering the difficulty of finding a measurable state variable of the device with physical meaning, we directly take the flux $\varphi(t)$ as the state variable, which can be easily calculated from the input $v(t) . R(\varphi(t), v(t))$ consists of $\mathrm{HH}$ functions (10). There are existing identification algorithms for $\mathrm{HH}$ functions, see [19], [26] for more information. The number $m$ of hinge functions and the parameters $a_{i}, b_{i}, c_{i}, i=1, \ldots, m$ in (10) are artificially selected. Then parameters $a_{0}, b_{0}, c_{0}$ and $\eta_{i}, i=1, \ldots, m$ are calculated by least squares method based on the experiment data.

In the gradual resistance tuning situation, pulses with $5 \mu$ s width and different amplitudes are applied to the AgInSbTe memristor device [8]. Voltage dependence is observed. Specifically, the resistance tuning speed and the range of ultimate resistance state is dependent on the amplitude of the voltage, see data plot in Fig. 1. The flux $\varphi(t)$ at the $n$th pulse is calculated by $\varphi(t)=n A_{v} \Delta_{v}$, where $A_{v}$ is the amplitude and $\Delta_{v}$ is the width of the pulse. Hinge functions in the form of $\max \left\{0, v+c_{i}\right\}$ are used to model the voltage dependence, and hinge functions in the form of $\max \left\{0, \varphi+c_{i}\right\}$ are used to characterize the nonlinear relationship of the flux and the resistance in the resistance tuning. Our model fits the experiment data well, as shown in Fig. 1.

In the voltage sweep situation, we use a different set of parameters in (10), since most of the sweep voltage $([-0.4 \mathrm{~V}, 0.4 \mathrm{~V}])$ is beyond the voltage range of the gradual resistance tuning situation $([-1.2 \mathrm{~V},-0.4 \mathrm{~V}]$ and
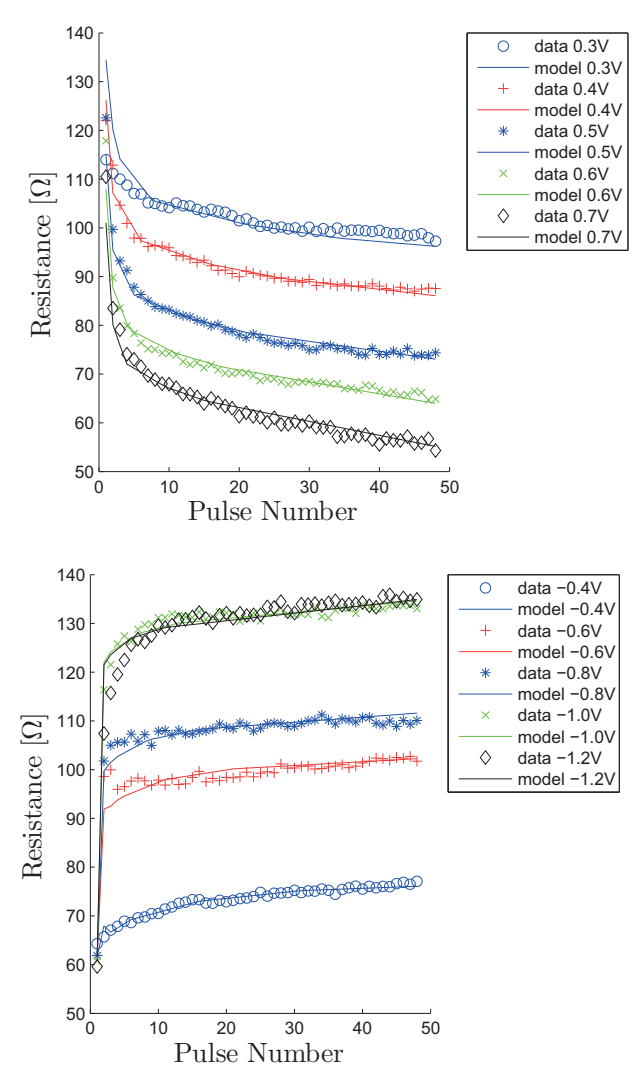

Fig. 1. Gradual resistance tuning situation. Pulses with $5 \mu \mathrm{s}$ width and different amplitudes are applied to the AgInSbTe memristor. The data are given in [8], and the lines are calculated from our model with the flux $\varphi$ and the voltage $v$ of each data point. 7 hinge functions are used for $v(t)>0$, and 8 hinge functions are used for $v(t)<0$.

$[0.4 \mathrm{~V}, 0.7 \mathrm{~V}])$. Considering the voltage dependence characteristic of the device, the parameters suitable for the gradual resistance tuning situation may be not suitable for a different range of the voltage. For example, $\forall v \in$ $[-0.4,0.4], \max \{0, v-0.4\}=0$, which means hinge function $\max \{0, v-0.4\}=0$ cannot characterize the voltage dependence in $[-0.4,0.4]$. Also the sweep response of the device may have different characteristics compared with the pulse response of the device. Our model fits the experiment data well, as shown in Fig. 2.

Besides the amplitude of the pulse, the pulse width also has influence on the gradual resistance tuning of the AgInSbTe memristor [8]. Even with the same amplitude of the pulse, different pulse widths will cause significantly different resistance tuning results, as shown by the data plots in Fig. 3. With a little modification, our model can also characterize the dependence of the pulse width in the unipolar gradual resistance tuning of the AgInSbTe memristor. The gradual resistance tuning of pulses with $-1 \mathrm{~V}$ amplitude and different widths is modeled. $\varphi(t)$ of different widths are separately calculated from $\varphi(0)=0$ for the model. Figure 3 shows that our model can characterize the dependence of the pulse width well. The data points are given in [8], and the lines are calculated from our model. 


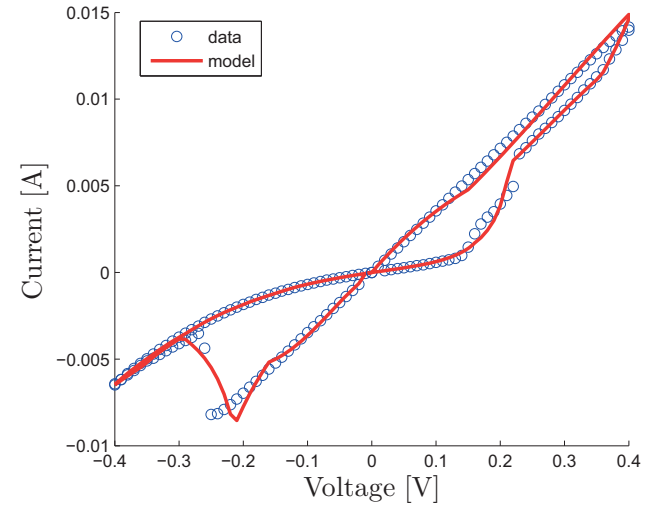

Fig. 2. Voltage sweep situation. Triangular sweep voltage is applied to the AgInSbTe memristor. The data is given in [8], and the lines are calculated from our model. 5 hinge functions are used for $v(t)>0$, and 8 hinge functions are used for $v(t)<0$.

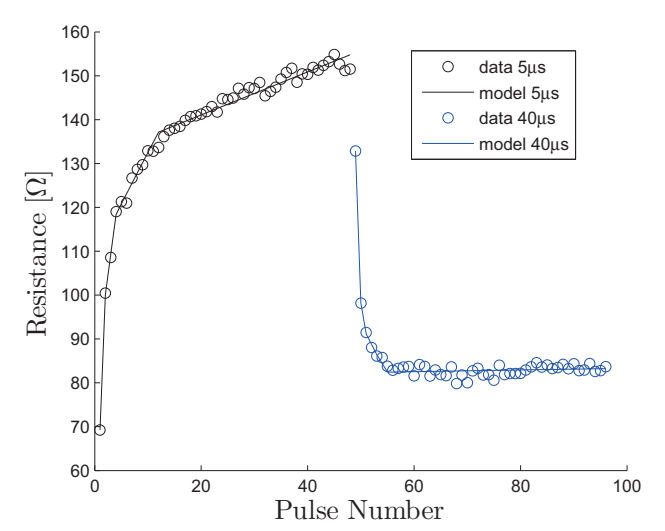

Fig. 3. Unipolar gradual resistance tuning situation. Pulses with $-1 \mathrm{~V}$ amplitude and different widths are applied to the AgInSbTe memristor. 9 hinge functions are used.

In the further studies, to take the amplitude and width of the pulse into account at the same time, we can use

$$
\begin{aligned}
& R(\varphi(t), v(t), \Delta) \\
= & a_{0} \varphi(t)+b_{0} v(t)+d_{0} \Delta+c_{0} \\
& +\sum_{i=1}^{m} \eta_{i} \max \left\{0, a_{i} \varphi(t)+b_{i} v(t)+d_{i} \Delta+c_{i}\right\} .
\end{aligned}
$$

The parameters in (12) can be determined by the same method we used in this section with data points in the form of $(\varphi, v, \Delta, R)$. Lack of physical meaning is a limitation of our model. If we are able to find a proper state variable $y$ with physical meaning in the further studies, the memristive model

$$
\begin{aligned}
i(t) & =\frac{1}{R(y(t), v(t))} v(t), \\
\frac{\mathrm{d} y(t)}{\mathrm{d} t} & =f(y(t), v(t)),
\end{aligned}
$$

can be more suitable to describe the AgInSbTe memristor. We can still use $R(y(t), v(t))$ and $f(y(t), v(t))$ that consist of $\mathrm{HH}$ functions $\max \left\{0, a_{i} y(t)+b_{i} v(t)+c_{i}\right\}$ to fit the experimental data.

\section{Discussion and Conclusion}

In this paper, we give a compact mathematical model of the AgInSbTe memristor based on experiment data for different kinds of input signals. With usage of the piecewise linear technique, which has strong approximation capability and effective identification algorithms, our flux-voltage controlled memristor model fits the experiment data well. The mathematical model can be used in simulation, analysis and further studies of circuits and systems consisting of the AgInSbTe memristor and other analog memristors, such as emulating biological plastic synapses [27], [28].

The flux-voltage controlled memristor model and the piecewise linear approximation method can be used to model other physical memristor devices with experiment data as well. Only experiment data of the voltage and the corresponding resistance is needed, and the flux can be calculated from the voltage. While for a general memristive system (5), if flux or charge is not used as the state variable, data and information of a particular state variable of the device with physical meaning and its derivative are required. The choice of such state variable usually requires a deep understanding of the physical mechanism of the device. Unlike the voltage, the data of such state variable and its derivative may be difficult to measure. This makes it not easy to get a general memristive system that approximates the device well. Besides the measurement of the state variable, the flux-voltage controlled memristor model may also simplify the calculation of the differential equation. For example, given input signal $v(t)=A \sin \omega t$, we can directly have $\varphi(t)=\int_{0}^{t} v(\tau) \mathrm{d} \tau=A(1-\cos \omega t) / \omega$. For a general memristive system (5), the state variable usually is calculated through numerical simulation, since the differential equation $\dot{x}=f(x, v, t)$ is hard to solve. Such simulation may be time consuming, and the simulation errors need to be considered [15], [29].

In this paper, we artificially select the hinge and use least squares method to calculate other parameters in the piecewise linear approximation of the flux-voltage controlled memristor model. There are also effective identification algorithms for $\mathrm{HH}$ functions [26], which can automatically select and adjust the hinge. Besides the $\mathrm{HH}$ functions, many other kinds of piecewise linear functions can also be used in the approximation [13], [20], [30]. All these piecewise linear functions are capable of approximating multivariable nonlinear function when modeling memristor devices.

For a general memristive system (5), the piecewise linear technique is still applicable. Any function can be approximated by a piecewise linear function [13], [19], [20]. The strong approximation capability ensures that the piecewise linear approximation of $f(x, v, t)$ and $G(x, v, t)$ can fit the data well. For example, with the HH functions, $f(x, v, t)$ can be represented as $f(x, v, t)=\sum_{i=1}^{m} \eta_{i} \max \left\{0, a_{i} x+b_{i} v+\right.$ $\left.d_{i} t+c_{i}\right\}$. 
The piecewise linear function is different from a general nonlinear function: the piecewise linear function is linear at each subregion of the domain, which allows linear methods to be used separately in each subregion. There are many methods specially designed for piecewise linear optimization and stability analysis, see [22], [23], [24]. With piecewise linear flux-voltage controlled memristor model, these methods can be applied to circuits and systems consisting of memristors.

\section{Acknowledgments}

This project is jointly supported by the National Natural Science Foundation of China (61473165, 61134012, 61376130), the National Basic Research Program of China (2012CB720505), the National High-Tech R\&D Program of China (2011AA010404), and the Fundamental Research Funds for the Central Universities (HUST: 0118182046).

\section{References}

[1] CHUA, L. O. Memristor - the missing circuit element. IEEE Transactions on Circuit Theory, 1971, vol. 18, no. 5, p. 507-519. ISSN: 0018-9324. DOI: 10.1109/TCT.1971.1083337

[2] CHUA, L. O. Resistance switching memories are memristors. Applied Physics A, 2011, vol. 102, no. 4, p. 765-783. ISSN: 0947-8396. DOI: $10.1007 / \mathrm{s} 00339-011-6264-9$

[3] CHUA, L. O., KANG, S. M. Memristive devices and systems. Proceedings of the IEEE, 1976, vol. 64, no. 2, p. 209-223. ISSN: 00189219. DOI: 10.1109/PROC.1976.10092

[4] DI VENTRA, M., PERSHIN, Y. V., CHUA, L. O. Circuit elements with memory: memristors, memcapacitors, and meminductors. Proceedings of the IEEE, 2009, vol. 97, no. 10, p. 1717-1724. ISSN: 0018-9219. DOI: 10.1109/JPROC.2009.2021077

[5] STRUKOV, D. B., SNIDER, G. S., STEWART, D. R., et al. The missing memristor found. Nature, 2008, vol. 453, no. 7191, p. 80-83. ISSN: 0028-0836. DOI: 10.1038/nature06932

[6] PERSHIN, Y. V., DI VENTRA, M. Spin memristive systems: spin memory effects in semiconductor spintronics. Physical Review $B$, 2008, vol. 78, no. 11, p. 113309. ISSN: 1098-0121. DOI: 10.1103/PhysRevB.78.113309

[7] StraChan, J. P., TORREZAN, A. C., MiAO, F., et al. State dynamics and modeling of tantalum oxide memristors. IEEE Transactions on Electron Devices, 2013, vol. 60, no. 7, p. 2194-2202. ISSN: 0018-9383. DOI: 10.1109/TED.2013.2264476

[8] ZHANG, J. J., SUN, H. J., LI, Y., et al. AgInSbTe memristor with gradual resistance tuning. Applied Physics Letters, 2013, vol. 102, no. 18 , p. 183513. ISSN: 0003-6951. DOI: 10.1063/1.4804983

[9] PICKETT, M. D., STRUKOV, D. B., BORGHETTI, J. L., et al. Switching dynamics in titanium dioxide memristive devices. Journal of Applied Physics, 2009, vol. 106, no. 7, p. 1-6. ISSN: 0021-8979. DOI: $10.1063 / 1.3236506$

[10] YAKOPCIC, C., TAHA, T. M., SUBRAMANYAM, G., et al. A memristor device model. IEEE Electron Device Letters, 2011, vol. 32, no. 10, p. 1436-1438. ISSN: 0741-3106. DOI: 10.1109/LED.2011.2163292
[11] KVATINSKY, S., FRIEDMAN, E. G., KOLODNY, A., et al. TEAM: threshold adaptive memristor model. IEEE Transactions on Circuits and Systems I: Regular Papers, 2013, vol. 60, no. 1, p. 211-221. ISSN: 1549-8328. DOI: 10.1109/TCSI.2012.2215714

[12] ASCOLI, A., CORINTO, F., SENGER, V., et al. Memristor model comparison. IEEE Circuits and Systems Magazine, 2013, vol. 13, no. 2, p. 89-105. ISSN: 1531-636X. DOI: 10.1109/MCAS.2013.2256272

[13] CHUA, L. O., DENG, A. C. Canonical piecewise-linear modeling. IEEE Transactions on Circuits and Systems, 1986, vol. 33, no. 5, p. 511-525. ISSN: 0098-4094. DOI: 10.1109/TCS.1986.1085952

[14] ITOH, M., CHUA, L. O. Memristor oscillators. International Journal of Bifurcation and Chaos, 2008, vol. 18, no. 11, p. 3183-3206. ISSN: 0218-1274. DOI: 10.1142/S0218127408022354

[15] YU, J., MU, X., XI, X., et al. A memristor model with piecewise window function. Radioengineering, 2013, vol. 22, no. 4, p. 969974. ISSN: $1210-2512$

[16] MA, C., XIE, S., JIA, Y., et al. Macromodeling of the memristor using piecewise Volterra series. Microelectronics Journal, 2014, vol. 45, no. 3, p. 325-329. ISSN: 0026-2692. DOI: 10.1016/j.mejo.2013.11.017

[17] MU, X., YU, J., WANG, S. Modeling the memristor with piecewise linear function. International Journal of Numerical Modelling: Electronic Networks, Devices and Fields, 2015, vol. 28, no. 1, p. 96-106. ISSN: 0894-3370. DOI: 10.1002/jnm.1987

[18] LIN, Z., WANG, H. Image encryption based on chaos with PWL memristor in Chua's circuit. In International Conference on Communications, Circuits and Systems. Chengdu (China), 2009, p. 964-968. ISBN: 978-1-4244-4886-9. DOI: 10.1109/ICCCAS.2009.5250354

[19] BREIMAN, L. Hinging hyperplanes for regression, classification, and function approximation. IEEE Transactions on Information Theory, 1993, vol. 39, no. 3, p. 999-1013. ISSN: 0018-9448. DOI: $10.1109 / 18.256506$

[20] WANG, S., HUANG, X., JUNAID, K. M. Configuration of continuous piecewise-linear neural networks. IEEE Transactions on $\mathrm{Neu}$ ral Networks, 2008, vol. 19, no. 8, p. 1431-1445. ISSN: 1045-9227. DOI: $10.1109 /$ TNN.2008.2000451

[21] MU, X., YU, J., WANG, S. Modeling the flux-charge relation of memristor with neural network of smooth hinge functions. Radioengineering, 2014, vol. 23, no. 3, p. 939-943. ISSN: 1210-2512

[22] VIELMA, J. P., AHMED, S., NEMHAUSER, G. Mixed-integer models for nonseparable piecewise-linear optimization: unifying framework and extensions. Operations Research, 2010, vol. 58, no. 2, p. 303-315. ISSN: 0030-364X. DOI: 10.1287/opre.1090.0721

[23] HUANG, X., XU, J., MU, X., et al. The hill detouring method for minimizing hinging hyperplanes functions. Computers \& Operations Research, 2012, vol. 39, no. 7, p. 1763-1770. ISSN: 0305-0548. DOI: 10.1016/j.cor.2011.10.017

[24] SUN, Z. Stability of piecewise linear systems revisited. Annual Reviews in Control, 2010, vol. 34, no. 2, p. 221-231. ISSN: 1367-5788. DOI: $10.1016 /$ j.arcontrol.2010.08.003

[25] LIU, M., ABID, Z., WANG, W., et al. Multilevel resistive switching with ionic and metallic filaments. Applied Physics Letters, 2009, vol. 94, no. 23, p. 233106-233106. ISSN: 0003-6951. DOI: $10.1063 / 1.3151822$

[26] PUCAR, P., SJOBERG, J. On the hinge-finding algorithm for hingeing hyperplanes. IEEE Transactions on Information Theory, 1998, vol. 44, no. 3, p. 1310-1319. ISSN: 0018-9448. DOI: $10.1109 / 18.669422$

[27] JO, S. H., CHANG, T., EBONG, I., et al. Nanoscale memristor device as synapse in neuromorphic systems. Nano letters, 2010, vol. 10, no. 4, p. 1297-1301. ISSN: 1530-6984. DOI: $10.1021 / \mathrm{nl} 904092 \mathrm{~h}$ 
[28] LI, Y., ZHONG, Y., XU, L., et al. Ultrafast synaptic events in a chalcogenide memristor. Scientific Reports, 2013, vol. 3, no. 1619, p. 1-7. ISSN: 2045-2322. DOI: 10.1038/srep01619

[29] BIOLEK, D., BIOLEK, Z., BIOLKOVA, V., KOLKA, Z. Modeling of TiO2 memristor: from analytic to numerical analyses. Semiconductor Science and Technology, 2014, vol. 29, no. 12, p. 125008. ISSN: 0268-1242. DOI: 10.1088/0268-1242/29/12/125008

[30] HUANG, X., MU, X., WANG, S. Continuous piecewise linear identification with moderate number of subregions. In 16th IFAC Symposium on System Identification. Brussels (Belgium), 2012, p. 535-540. DOI: 10.3182/20120711-3-BE-2027.00251

\section{About the Authors...}

Juntang YU received the B.S. degree in Control Science and Engineering from Harbin Institute of Technology, Harbin, China in 2011. He is currently pursuing the Ph.D. degree from the Department of Automation, Tsinghua University, Beijing, China. His current research interests lie in the area of modeling and analysis of memristor circuits and systems.

Yi LI received the B.S. degree in Electronic Science and Technology, Huazhong University of Science and Technology, China in 2009. Currently he is working towards the Ph.D. degree at the School of Optical and Electronic Information, Huazhong University of Science and Technology, China. His current research interests include phase change memory and memristive devices for neurmorphic applications.

Xiaomu MU received the B.S. degree in control science and engineering from Tsinghua University, Beijing, China in 2009. He is currently a Ph.D. candidate in the Department of Automation, Tsinghua University, Beijing, China. His current research areas include nonlinear dynamic system control, piecewise linear identification and memristor device.
Jinjian ZHANG received the B.S. degree in Electronic Science and Technology, Huazhong University of Science and Technology, China in 2010. Currently he is working towards the Ph.D. degree at the School of Optical and Electronic Information, Huazhong University of Science and Technology, China. His current research interests focus on the memristive materials and their applications.

Xiangshui MIAO received the B.S. degree, M.S. degree and the Ph.D. degree in Electronic Materials and Devices from Huazhong University of Science and Technology, China, in 1986, 1989, and 1996. He was an Associate Research Fellow from 1996 to 1997 in the Department of Electronic Engineering, City University of Hong Kong, in 1997. He was a Senior Scientist from 1997 to 2007 in Data Storage Institute, Singapore. Since 2007 he has been a Full Professor in School of Optical and Electronic Information, Huazhong University of Science and Technology, China. His current research interests cover the fields of novel information storage materials and devices, including phase change memory, memristor, artificial cognitive memory.

Shuning WANG received the B.S. degree in Electrical Engineering from Hunan University, China in 1982, the M.S. degree and the Ph.D. degree both in the system engineering from Huazhong University of Science and Technology, China in 1984 and 1998, respectively. He was an Associate Professor from 1992 to 1993 and a Full Professor from 1994 to 1995, at the Institute of Systems Engineering, Huazhong University of Science and Technology. He joined Tsinghua University, Beijing, China in 1996. Since then, he has been a Full Professor in Department of Automation, Tsinghua University. He was a Visiting Scholar in the College of Engineering, University of California at Riverside in 1994, and a Visiting Fellow in Department of Electrical Engineering, Yale University from 2001 to 2002. His current research interests are mainly in developing practical methods for nonlinear system identification, control and optimization via piecewise-linear approximation. 